\title{
Rice Leaf Disease Recognition using Local Threshold Based Segmentation and Deep CNN
}

\author{
Anam Islam, Redoun Islam, S. M. Rafizul Haque, S.M. Mohidul Islam \\ Computer Science and Engineering Discipline, Khulna University, Khulna, Bangladesh \\ E-mail: \{anamislam000, abir.cse.ku\}@gmail.com, \{rafizul, mohid $\} @$ cse.ku.ac.bd \\ Mohammad Ashik Iqbal Khan \\ Bangladesh Rice Research Institute, Gazipur, Bangladesh \\ E-mail: ashikjp@gmail.com
}

Received: 14 April 2021; Accepted: 23 June 2021; Published: 08 October 2021

\begin{abstract}
Timely detection of rice diseases can help farmers to take necessary action and thus reducing the yield loss substantially. Automatic recognition of rice diseases from the rice leaf images using computer vision and machine learning can be beneficial over the manual method of disease recognition through visual inspection. During the recent years, deep learning, a very popular and efficient machine learning algorithm, has shown great promise in image classification task. In this paper, a segmentation-based method using deep neural network for classifying rice diseases from leaf images has been proposed. Disease-affected regions of the rice leaves have been segmented using local segmentation method and the Convolutional Neural Network (CNN) has been trained with those images. Proposed method has been applied on three different datasets including the one created by us which consists of the rice leaf images collected from Bangladesh Rice Research Institute (BRRI). Three state-of-the-art CNN architectures VGG, ResNet and DenseNet, used in the proposed method, have been trained with these three datasets for classifying the diseases. Classification performance of the proposed method using the said three CNN architectures for the three datasets have been analyzed and compared. These results show that this model is quite promising in classifying rice leaf diseases. Outcome of this research is an enhancement in the performance of rice disease classification which is quite significant for the viability of this work to be transformed into a real-time application for the farmers.
\end{abstract}

Index Terms: Convolutional Neural Network, VGG16, ResNet50, DenseNet121, Local threshold, Rice leaf disease.

\section{Introduction}

Rice is one of the world's three main crops. Human consumption accounts for $78 \%$ of the total production of rice. Rice is the staple food of more than half of the global population - more than 3.5 billion people depend on rice for more than $20 \%$ of their daily calories. Asia accounts for $90 \%$ of global rice devouring, and total rice demand is emerging there. But outside Asia, where rice is not a staple yet, per capita consumption continues to grow. Rice is rapidly growing staple food in Africa, and one of the fastest in Latin America. Hence, trend of global rice consumption remains strong, driven by both population and economic growth, especially in many Asian and African countries [4]. In Bangladesh, it is the staple food of about 135 million people [5].

Despite being one of the most essential agricultural crops, rice is greatly affected by various diseases. Rice disease destroys 10-15\% of production in Asia [12]. Farmers lose an estimated average of $37 \%$ of their rice crop every year due to various types of rice diseases and pesticides [5]. Altogether, there are 32 rice diseases found in Bangladesh. Those different diseases are Rice Leaf Blast (RB), Bacterial Leaf Blight (BLB), Sheath Blight (SB), Brown Sport (BS), Tungro, Narrow Brown Spot (NBS) and Rice Neck Blast (RNB) etc. Among these diseases, rice blast is the most devastating.

In today's digital age, it is essential for the farmers to use the modern technology for efficient management of their crops. In Bangladesh, rice is cultivated twice in a year. Most of the farmers face many problems while cultivating rice due to the attack by fungi, bacteria, and viruses. For many cases, farmers are unable to identify the diseases correctly in time which results in the loss of expected yield. In Bangladesh, the production loss due to major diseases is presented in Table 1 [1] [2] [3]. 
Table 1. Production Loss due to Major Diseases in Bangladesh [1] [2] [3]

\begin{tabular}{|c|c|c|}
\hline No & Disease Name & Production Loss \\
\hline 1 & Rice Leaf Blast & $80 \%[2]$ \\
\hline 2 & Bacterial Leaf Blight & $30 \%[1]$ \\
\hline 3 & Sheath Blight & $30 \%[3]$ \\
\hline
\end{tabular}

When a rice disease outbreaks somewhere, agriculture scientists are engaged to investigate about the disease, and suggest proper advice to the farmers. But for limited number of scientists, sometimes it becomes difficult for them to cover the entire disease-affected region for visual inspection of the disease. As a result, farmers cannot recognize the disease and respond accordingly in a timely manner. Nowadays farmers can capture the images of the disease-affected rice leaves with cellphones. So, if images of these disease-affected leaves can be used to recognize the diseases, farmers need not to wait for the experts to visit their fields and can act promptly for the cure of the diseases. For this, technique for recognizing diseases from the images of the disease-affected leaves needs to be made available for the farmers in the rural areas. Hence, objective of this research is to classify rice diseases from the images of the disease-affected leaves where a cellphone should be good enough to capture the images.

Many studies have already been conducted to recognize the rice diseases. Most of these studies have used machine learning algorithms like support vector machine, genetic algorithm where features are extracted and are used to classify different diseases. The performance of convolutional neural network $(\mathrm{CNN})$ in image classification has recently been very promising, leading researchers to consider using this technique to identify rice diseases as well. This study has applied convolutional neural network for classifying rice diseases from the images. Convolutional neural network can extract the features itself which ensures no important features get missed during the feature extraction process. This is the advantage of this method that it can save the cost of extracting the features which is computationally very expensive.

The rest of the paper is organized as follows. In the next section, some works related to our study have been discussed. The proposed method for the recognition of rice diseases has been explained in section three. The experimental results of our proposed method using different datasets are presented in section four. In the final section, concluding remarks and scope for further improvement are stated in brief.

\section{Related Works}

Plant disease detection methods, during the early years, were based on using general image processing techniques. Support vector machine, K-means clustering, genetic algorithms were used as the classifiers in these studies.

In ref. [24], authors have proposed a method to detect rice disease using Support Vector Machine. Rice Bacterial Leaf Blight (BLB), Rice Sheath Blight (SB) and Rice Blast (RB) have been classified successfully with an accuracy of $97.2 \%$. Their method applied general approaches in image acquisition, resizing and noise reduction. Otsu's method is used for segmentation with a modified threshold value. Shape and color texture features were applied in the process. Finally, using these features, disease classification was performed using support vector machine (SVM). In this experiment, a sample of seventy-two disease spots was used for each disease. Three models were designed. One with shape and texture feature, one with only texture and another with only shape. The first model produced better results than the others. This work has used disease spot as the only input; however, the number of spots and how they are spread could also be useful to recognize disease more accurately.

A study on the classification of rice diseases and the prediction of its extent has been presented in [17]. Their method can be used for diagnosis of three common rice diseases, Brown Spot, Leaf Blast and Bacterial Leaf Blight. To separate the disease-affected part, K-Means Clustering method is used. Color, texture, and shape features were extracted from the disease-affected parts of the images. Rice diseases are recognized using SVM classifier and the suggestions on the pesticides and fertilizers are provided according to the disease stage. This method has a accuracy of $90.95 \%$ for Brown Spot, $94.11 \%$ for Leaf Blast and $85.71 \%$ for Bacterial Leaf Blight. Although this work achieved good accuracy, it recognized only three diseases.

Prajapati et al. has proposed a method of detection and classification of rice diseases in [18]. They have introduced a background removal technique which works fine for UCI Machine Learning Repository dataset. Then they applied Kmeans clustering to segment the disease-affected spots from the leaf images. They extracted 88 features from the segmented images and obtained $100 \%$ training accuracy and $73.33 \%$ test accuracy. Here, accuracy is relatively low than that of other works.

Probabilistic Neural Network (PNN) has been used for rice disease detection in [10]. In this paper, authors attempted to identify four major rice diseases using fractal descriptors to analyze the texture of the lesions. They focused on descripting the color and texture of the disease only. The disease affected leaf images were collected and lesions were cropped manually. Each of these images was converted into HSV color space. Histogram was built and Laplacian filter was used in image sharpening. Then fractal descriptors were extracted from the lesion using Fourier spectrum. For disease classification, they used probabilistic neural network. This study achieved promising performance in detecting Brown Spot, Bacterial Leaf Blight and Tungro with an accuracy of 92.31\%, 96.25\% and 97.96\% 
respectively. The accuracy dropped to $83.00 \%$ while detecting Leaf Blast. This work did not apply any shape feature which could be useful to recognize the diseases and accordingly could improve the accuracy.

A new approach for developing plant disease recognition model using deep convolutional neural network has been proposed in [23]. This study used 4500 images for the experiment. The images were preprocessed by cropping manually and resized to reduce the time for training. All the images were augmented using offline transformation, perspective transformation and rotation. A modified CaffeNet architecture was used for training. The model was trained with 30880 images. Also 2589 images were used for validation. Fine tuning was performed to improve the efficiency. Using the proposed method, achieved accuracy was $95.8 \%$ which was boosted to $96.3 \%$ with fine-tuning. Dataset used in this study is imbalanced i.e., some classes have few images, and some other have very large number of images, which potentially contributed to the differences in the achieved accuracies for each class.

Fungal diseases of commercial crops like chili, cotton and sugarcane were detected in a study by Pujari et. al [22]. Discrete wavelet transform (DWT) was applied for feature extraction, whereas principal wavelet analysis was used for further feature reduction. Statistical based Mahalanobis distance and Probabilistic neural network (PNN) were used as classifiers. Average classification accuracies for the said classifiers were $83.17 \%$ and $86.48 \%$ respectively.

Singh et. el have used bag of visual words to develop a plant disease detection system [19]. A total of 536 affected and unaffected leaf images of mango, lemon, eucalyptus, neem, and Indian beech were used for this study. Features were extracted from the images and SVM was used to classify the diseases. Accuracy, sensitivity, and specificity were the metrics to evaluate the model.

In this study [9], a method to detect and analyze late blight disease from tomato leaf images has been proposed. A segmentation method has been applied which can extract the diseased spots from a leaf image. These segmented images are then analyzed using K-means clustering to identify late blight disease. This work has achieved $84 \%$ detection accuracy.

Some works have applied convolutional neural network to classify diseases using the diseased leaves. In ref. [14], authors used convolutional neural network to detect disease from apple leaves. The neural network was trained with 21,794 images. These images were preprocessed and augmented on direction and light disturbance. This model was inspired by the classical AlexNet and GoogLeNet. The accuracy they achieved was $97.62 \%$ on their test set.

The ref. [21], was on tomato disease detection using leaf images. They used 17929 images of nine different diseases and healthy leaves. The diseases are Bacterial Spot, Early Blight, Late Blight, Leaf Mold, Septoria Leaf Spot, Spider Mite, Target Spot, Tomato Mosaic Virus and Yellow Leaf Curl Virus. They tested two methods, finding that the S-CNN model performed better with segmented image data than the F-CNN model trained with the entire image.

Deep convolutional neural network has been used for rice disease detection in [15]. There were three classes in the dataset including normal, diseased and snail infected rice plant. Their dataset contained only 227 images collected from the rice fields of the nearby districts. The authors used transfer learning on AlexNet which is a relatively small scale and old CNN model. They used various image augmentation techniques on these collected images and managed to get test accuracy of $91.23 \%$. During the classification task, this method did not consider classifying the specific disease of the affected rice plant. The model can only tell if the plant is affected by disease or not.

In ref. [13], the authors have proposed a novel rice blast recognition method. A dataset of 2906 positive and 2902 negative samples was provided for training and testing CNN model and achieved 95.83\% accuracy. They also conducted comparative experiments for different models, which resulted in the favor of high-level features extracted by CNN than handcrafted features like local binary pattern histograms (LBPH) and wavelet transform (Haar-WT). As a result, they concluded that their CNN model outperforms other approaches in detecting rice blast disease. This work is not suitable to be used practically for various diseases as it only recognizes rice blast disease which is a major limitation.

A deep learning technique to recognize rice diseases has been proposed in [20]. A total of 1426 images of different types of rice diseases have been collected and used in the models utilizing VGG16 and InceptionV3. A small two staged CNN model for mobile devices also has been proposed. This work achieved $94.7 \%$ accuracy which is pretty good though the images were collected from the same source; accuracy for images collected from different sources could justify the consistency in the classification performance of this work.

In ref. [11], CNN with transfer learning has been applied to classify three types of rice diseases Leaf Blight, Rice Blast and Brown Spot, along with healthy leaves. The dataset contains 1509 in total and achieved accuracy is $92.46 \%$ for transfer learning. This work could be more efficient by using brightness augmentation in preprocessing.

$\mathrm{Al}$-amin M. et al. [7] used 900 images to classify four types of diseases. They applied gray scaling, blur removal and segmented the background for every image. Then with CNN, they achieved $97.4 \%$ accuracy. Though the dataset in this study has a fairly good number of images for four types of diseases, it did not use augmentation which could enhance the efficacy of the method.

FCM-KM and faster R-CNN fusion was proposed in the study by Zhou G. et al. [25] to classify rice diseases using a dataset of 3010 images. The method uses two-dimensional filtering mask for noise reduction and two-dimensional Otsu threshold segmentation method to reduce background interference. Then, K-means clustering was used to determine the optimal clustering $\mathrm{k}$ value. Then with R-CNN, FCM-KM analysis was used to detect the rice diseases. Accuracies achieved for disease rice blast, bacterial blight and blight were $96.71 \%, 97.53 \%$ and $98.26 \%$ respectively.

In a study by Mohapatra D. et al. [16], CNN has been used to classify rice diseases, but the authors did not mention 
anything about the number of images in the dataset and the achieved accuracy; rather, they provided some input images and how accurately the method can identify the disease.

\section{Materials and Methods}

A novel dataset of our own containing images of both disease-affected and healthy rice leaves, acquired from different sources, and two other freely available datasets have been used in this study. Evaluating performance of our proposed method for three different datasets made it possible to justify the consistency and efficacy of the method. Some preprocessing steps like background removing and resizing were applied on these images. Then, image segmentation was performed to extract the disease-affected spots. These images were used as the input into the convolutional neural network $(\mathrm{CNN})$ based classifier algorithm. Image augmentation technique has been used to increase the number of images during the training phase. The images of validation and test sets were also preprocessed similarly. These preprocessing steps have been useful to make the images analogous in several aspects and thus suitable to be processed by CNN. Selection of CNN as the classifier algorithm has eliminated the need for feature extraction, which is not only computationally expensive but also selecting proper set of features is not simple. Hence, use of three different datasets along with the stated methods eventually resulted in achieving satisfactory classification performance. Workflow diagram of the proposed method is shown in Fig. 1.

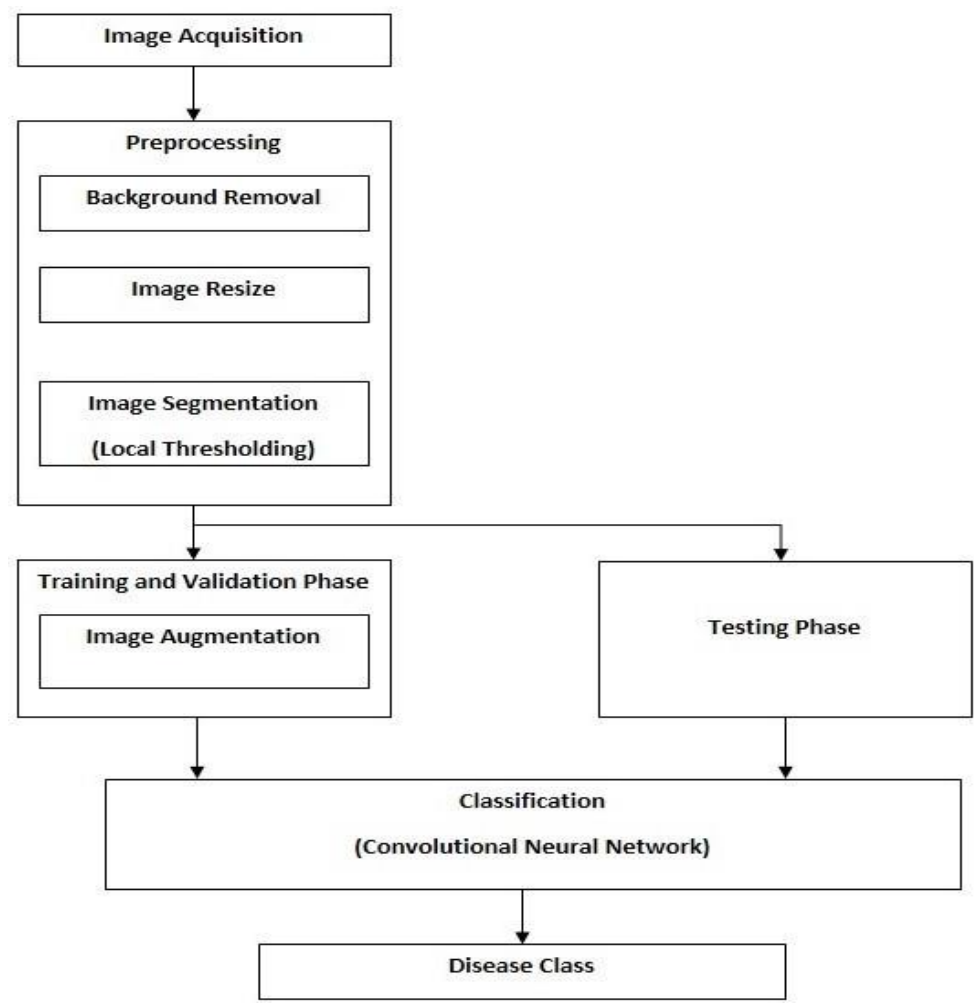

Fig.1. Workflow diagram of the proposed method.

\subsection{Dataset}

We collected three separate datasets for this work. One of them was created by us with a collection of five different diseases and also healthy leaf. Other two were collected from different sources. We used $60 \%$ data to train our classifier algorithm, $20 \%$ data was used for validation and the rest $20 \%$ was used for testing.

\section{A. Creation of our own Dataset}

A novel dataset has been created for this study by capturing images from the fields of Bangladesh Rice Research Institute (BRRI). All the images are RGB with diverse backgrounds. The images were captured in proper light. Digital camera and cell phone camera were used for capturing these images. The resolution of the images is not same for all. Some of the sample images are shown in Fig. 2. The experts of BRRI have assisted us to capture the images of five different classes and label them properly. The classes are (a) Bacterial Leaf Blight, (b) Leaf Blust, (c) Sheath Blight, (d) Tungro and (e) Healthy Leaf. A total of 386 images were captured where 82 were of Bacterial Leaf Blight affected, 79 were of Leaf Blust affected, 82 were Sheath Blight affected, 73 were Tungro affected and 70 were healthy leaves. Most of these images were captured in sunlight. All the images of the dataset are in .jpg format. 


\section{B. Two more Datasets}

In addition to our own dataset, two more datasets have been used for evaluating the performance of the proposed method. One of the datasets is from UCI Machine Learning Repository [6] and the other one is from GitHub [8].

UCI Machine Learning Repository Dataset: A dataset available in UCI Machine Learning Repository [6] contains a set of 120 images consisting of three types of diseases. These are, (a) Bacterial Leaf Blight, (b) Brown Spot and (c) Leaf Smut. Each class contains 40 images of disease-affected leaves. This dataset was made by manually splitting the affected leaves into different disease classes. The disease name of the sample image was confirmed by consulting with the agriculture experts. The images were captured with a white background in direct sunlight. Then it was reduced to the desired resolution for processing.

GitHub Dataset: This dataset [8] was created for an Image Recognition application. This dataset contains 278 images of three diseases, of which 96 are Bacterial Leaf Blight, 102 are Brown spot and 80 images are of Leaf Blast. All the disease-affected leaves that were captured as images in the datasets were of optimum age ( 3 to 4 weeks). Most of the images contain heterogeneous background.

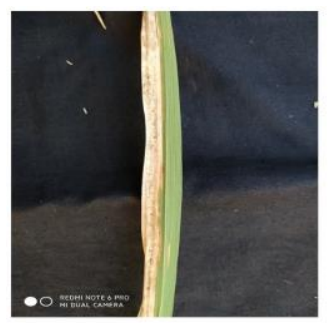

(a)

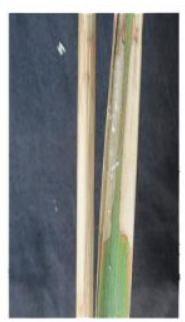

(d)

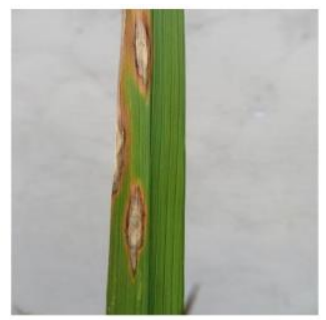

(b)

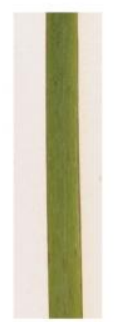

(e)

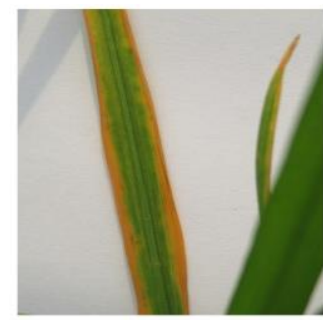

(c)

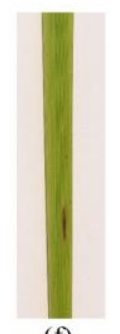

(f)

Fig.2. Collected Images of rice plant diseases (a) Bacterial Leaf Blight, (b) Leaf Blast, (c) Tungro, (d) Sheath Blight, (e) Leaf Smut and (f) Brown Spot.

\subsection{Image Preprocessing}

The goal of this step is to make the images ready for the machine learning model to make it easier to analyze and process computationally. The acquired images are usually messy and come from different sources. To feed them to the machine learning model, they need to be standardized and cleaned up. Preprocessing is usually used to perform steps that reduce the complexity and improve the results of the applied algorithm. Following preprocessing steps performed on the images of the dataset are described in this here.

\section{A. Background Removal}

In image preprocessing, the first task is background removal. When field images and other dataset images were captured and collected, their background was composed of human hands, soils, rice plants, and other objects. Different background removal algorithms were applied but we could not find a generalized algorithm that works perfectly for all the images. This kind of heterogeneous background makes the segmentation task quite difficult to extract important features. To overcome this problem, the unnecessary background of all the images was removed manually. We used Adobe Photoshop cc 2018 for background removal. First, the leaf parts of the images were selected with the Photoshop's built in selection tool. After selecting the leaf, we deleted the background and put the left in a gray colored background. No resizing was done in this scenario. Fig.3 and Fig.4 show the images before and after removing background respectively. 


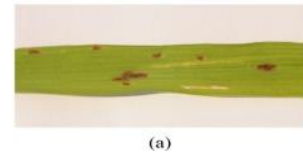

(a)

Fig.3. Images before removing background.

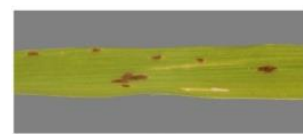

(a)

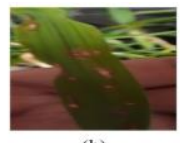

(b)

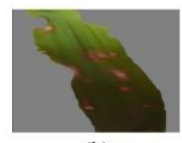

(b)

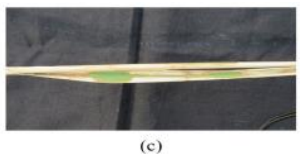

(c)

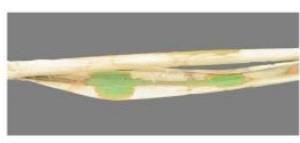

Fig.4. Images after removing background.

\section{B. Resize}

After removing the background, images were resized. High-definition cameras were used for capturing the images. As a result, the resolution of the images was very high. That's why, size of the images needed to be reduced. Moreover, the images were collected from multiple sources. So, the images were of different sizes. To get better result, converting all the images to the same size became essential. So, the images were resized to $300 \times 300$.

\subsection{Image Segmentation}

The goal of segmentation is to simplify and change the representation of an image into something more meaningful and easier to analyze.

Different types of methods for segmentation are used for various tasks. Generally, threshold methods are used in detection of leaf disease. For thresholding, specifically, Otsu's thresholding is useful, as it automatically calculates the threshold value.

Local threshold segmentation is used to segment the images for separating diseased spots. It can be observed that the disease-affected spots are covered with red and brownish edges [24]. So first, the images were thresholded based on the value of the red channel compared to the other two channels. If the red plane value is significantly larger than the green and blue plane value of the pixel, then they were considered as disease-affected spots.

After this thresholding, most of the disease-affected spots were obtained as white pixel values and other parts as black. But still, there were some holes in the image which could not be detected as the disease-affected spots. We had to carefully connect those to the disease-affected spots. To do that properly, first, the images were inverted and then the smaller components of the binary images whose areas were less than 200 pixels were removed. By following these steps, small regions of the disease-affected spots were acquired which were not detected through the threshold method. Then the images were inverted, and median filter was applied to remove noise.

In this way, we have now got a segmented binary image of the disease-affected parts. Finally, pixel values of the disease-affected regions were replaced with the original ones and thus we got our fully segmented disease-affected image. Algorithm 1 shows the segmentation algorithm used and Fig.5 shows the output of every step of segmentation process.

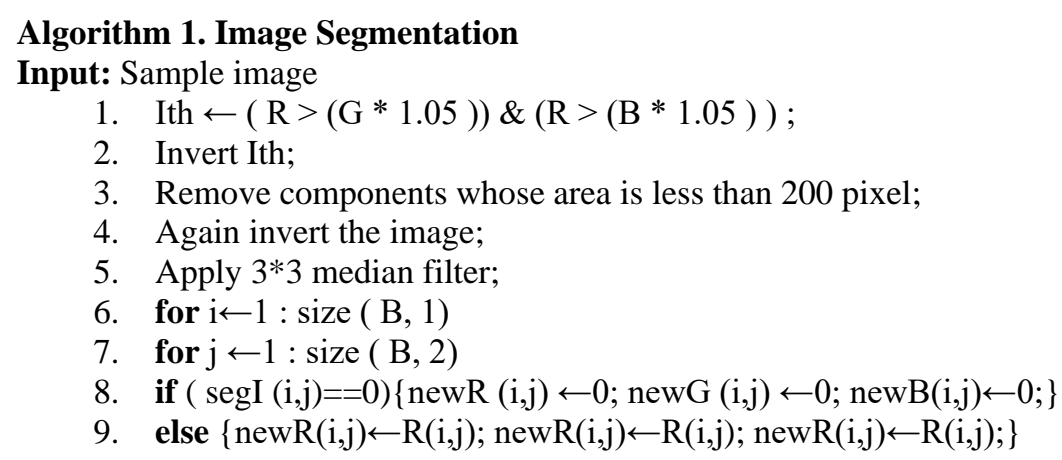



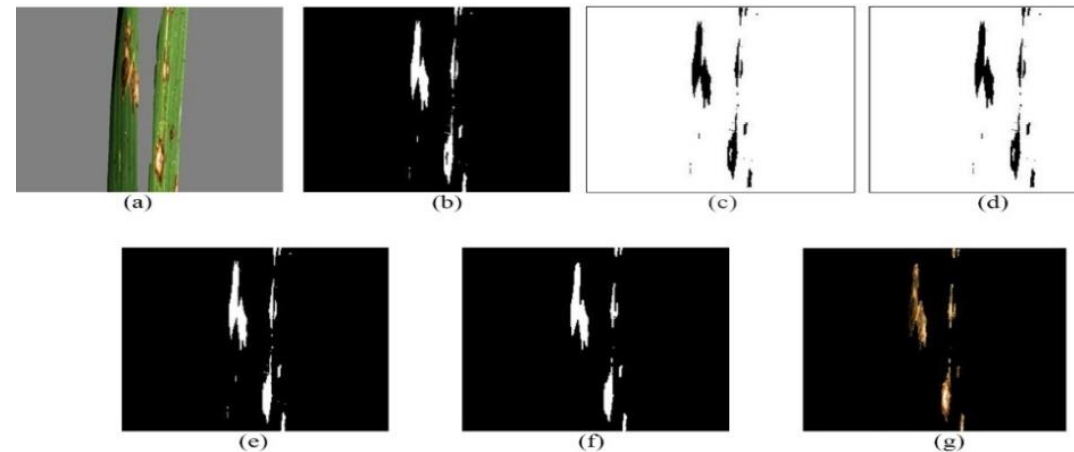

Fig.5. Output of every step of image segmentation (Leaf Blast) (a) Input image, (b) Threshold image, (c) Complement of threshold image, (d) After removing the smaller components from the image, (e) Again complemented the image, (f) After applying a median filter and (g) Finally output of the threshold image.

\subsection{Convolutional Neural Network Architecture}

In this study, three CNN architecture models, VGG16, Resnet50 and Densenet121 are used. VGG16 has 16 learning layers, Resnet50 has 50 convolution layers and DenseNet121 has 121 convolutional layers. Transfer learning and fine-tuning are applied in these models by removing the top layer of the models. Then two densely connected layers with 1000 and 500 nodes are added respectively with rectified linear unit (relu) activation.

$$
\operatorname{Relu}(X)=\max (0, X)
$$

Relu ensures nonnegative output. The final layer is softmax activation function. The layer contains same number of node as the number of class.

$$
\operatorname{Softmax}(x)=\frac{e^{z_{i}}}{\sum_{j=1}^{N} e^{z_{j}}}
$$

\section{Algorithm 2. Classifier Training}

Input: Sample segmented images

1. for $\mathrm{i} \leftarrow 1$ : total number of epochs

2. take image from training dataset

3. Calculate with the weight to get softmax outcome

4. Calculate loss with categorical cross entropy

5. Use optimizer to get the value to adjust weight

6. Adjust weights by back propagation.

7. Use validation data set to get the new accuracy

8. if (new accuracy $>$ previous best accuracy) best accuracy $=$ new accuracy

9. save new weights

So, when the training images were passed to the convolutional neural network, it gives the probabilistic values of the classes that the image could belong to. Using these values loss function is calculated and with that loss function value and learning rate, optimizer adjusts the weights of the neural network. below.

There are some parameters in convolutional neural network that can be tuned for better results. These are described

\section{A. Loss Function}

In this study categorical cross entropy has been used as loss function. This loss function is a log loss version of multi-class case. The log loss function is:

$$
-\sum_{c=1}^{N} y_{o, c} \log \left(p_{o, c}\right)
$$

Here, $N=$ number of classes, $\boldsymbol{y}_{\boldsymbol{o}, \boldsymbol{c}}=$ binary indicator $(0$ or 1$)$ if class level $c$ is the correct classification for observation, $o$. If the sample is actually form class 1 , then $\boldsymbol{y}_{\boldsymbol{o}, \boldsymbol{c}}$ will be 1 , only for $c=1$.

$\boldsymbol{p}_{\boldsymbol{o}, \boldsymbol{c}}=$ predicted probability if the observation, $o$ is actually from class c. suppose the observation, $o$ is from class Bacterial Leaf Blight. The determined probability for class Bacterial Leaf Blight is 1 . So, the loss will be none. So, the model correctly classified its sample. Again, if the probability is close to 0 , the loss value will be huge. It will give the 
output of a large positive number as the model makes completely opposite probabilistic assumption than what the assumption should be. So, the criterion is fulfilled through the loss function as well.

\section{B. Optimizer}

Adaptive Moment Estimation (Adam) is used in training our model. It is an extension to stochastic gradient descent $(s g d)$. The stochastic gradient descent maintains a predefined learning rate for all weight updates. Also, the learning rate does not change during training. But in Adam the learning rate changes separately. It has combined the advantages of two other extensions of stochastic gradient descents, which are,

(i) Adaptive Gradient Algorithm (AdaGrad): AdaGrad preserves a pre-parameter learning rate. It enhances performance on problems with sparse gradients.

(ii) Root Mean Square Propagation (RMSProp): RMSProp also preserves a pre-parameter learning rate. These are upgraded based on the mean of the recent magnitudes of the gradients for the weight.

Adam adapts the benefits of both. Rather than adapting the parameter learning rate based on the mean first moment as RMSProp, Adam also makes use of the mean of the second moments of the gradients, which is the uncentered variance.

\subsection{Training and Validation Phase}

Various types of disease-affected rice leaves were used in training the model. The steps are described below.

\section{A. Augmentation}

A large amount of training data is needed for a deep neural network to A large amount of training data is needed for a deep neural network to achieve good performance. To build a powerful and robust image classifier with the help of a very short training data, image augmentation is usually required for boosting the performance of a deep network. Image augmentation takes an image and artificially increases the number of images by applying a single or different kind of processing, such as random rotation, shifts, shear, and flips, etc.

Also, the model had to be trained in such a way that, it can handle input with all kinds of variations. We have applied zooming, brightness, shear, rotation, width shift, height shift and horizontal flip on our image for augmentation.

\subsection{Testing Phase}

As mentioned earlier, dataset is split into three parts, training, validation, and testing. Every set contains unique images of disease-affected leaf images. The training set is used to fit the model. The validation set gives an unbiased judgment of the model to fit on the training dataset while tuning model hyper parameters. The test set is kept fully separated from the training and validation sets to ensure the reliability of the achieved test accuracy. After training, it is used to get an unbiased evaluation of the model.

In this model, classification accuracy has been used as performance metric which is defined as the proportion of correct classifications to the total number of samples classified. The model weights were saved based on validation accuracy, which means, after every epoch, if the validation accuracy is better than the previous best one, then the updated weights are saved. If the validation accuracy does not give better result, the previous best one is kept. After completing all the epochs, the weights which performed best on validation set is used for future works and considered as the final weights of the model.

\section{Results}

Experimental results of the proposed method with relevant analysis are described in this section. This proposed method has been applied on three datasets including our own dataset, UCI Machine Learning Repository [6] and GitHub [8], as described in section 3.1. Three CNN models VGG16, ResNet50 and DenseNet121 have been applied to train the classification models for the said three datasets. The classification performance, in terms of validation accuracy and test accuracy, of the proposed method for the three said datasets for the three said CNN models are presented in Table 2. To know the full potential of the model, we have applied hold-out and 10-fold cross-validation on the datasets and the results are presented in Table 3. 
Table 2. Classification Accuracies for Different State of the art CNN Architectures

\begin{tabular}{|c|r|r|r|r|r|r|}
\hline & \multicolumn{2}{|c|}{ VGG16 } & \multicolumn{2}{c|}{ ResNet50 } & \multicolumn{2}{c|}{ DenseNet121 } \\
\cline { 2 - 7 } & $\begin{array}{c}\text { Validation } \\
\text { Accuracy }\end{array}$ & $\begin{array}{c}\text { Test } \\
\text { Accuracy }\end{array}$ & $\begin{array}{c}\text { Validation } \\
\text { Accuracy }\end{array}$ & $\begin{array}{c}\text { Test } \\
\text { Accuracy }\end{array}$ & $\begin{array}{c}\text { Validation } \\
\text { Accuracy }\end{array}$ & $\begin{array}{c}\text { Test } \\
\text { Accuracy }\end{array}$ \\
\hline $\begin{array}{c}\text { UCI Machine Learning } \\
\text { Repository Dataset }\end{array}$ & $85.94 \%$ & $83.33 \%$ & $33.33 \%$ & $33.33 \%$ & $94.64 \%$ & $91.67 \%$ \\
\hline GitHub Dataset & $83.00 \%$ & $72.72 \%$ & $40.00 \%$ & $36.36 \%$ & $90.91 \%$ & $85.45 \%$ \\
\hline Our Dataset & $77.46 \%$ & $80.59 \%$ & $45.83 \%$ & $23.63 \%$ & $90.63 \%$ & $89.85 \%$ \\
\hline
\end{tabular}

Table 3. Hold-out vs. 10-fold Cross-validation on the Datasets

\begin{tabular}{|c|c|c|c|c|}
\hline \multirow{2}{*}{} & \multicolumn{2}{|c|}{ DenseNet121 10 Fold } & \multicolumn{2}{c|}{ Densenet121 Holdout } \\
\cline { 2 - 5 } & $\begin{array}{c}\text { Validation } \\
\text { Accuracy }\end{array}$ & Test Accuracy & $\begin{array}{c}\text { Validation } \\
\text { Accuracy }\end{array}$ & Test Accuracy \\
\hline $\begin{array}{c}\text { UCI Machine Learning } \\
\text { Repository Dataset }\end{array}$ & $91.96 \%$ & $88.33 \%$ & $94.64 \%$ & $91.67 \%$ \\
\hline GitHub Dataset & $96.30 \%$ & $86.20 \%$ & $90.91 \%$ & $85.45 \%$ \\
\hline
\end{tabular}

Here, accuracies achieved for ResNet50 are poor for all three datasets. It is because of its shortcut connections. This property of ResNet50 is not suitable for this type of classification problem.

Table 4. Segmented and Non-segmented Dataset Accuracy

\begin{tabular}{|c|c|c|}
\hline & Validation Accuracy & Test Accuracy \\
\hline Segmented dataset & $94.64 \%$ & $91.67 \%$ \\
\hline Non Segmented Dataset & $91.99 \%$ & $83.33 \%$ \\
\hline
\end{tabular}

Table 5. Different Works in UCI Machine Learning Repository and their Test Accuracy

\begin{tabular}{|c|c|c|}
\hline & Segmentation Method & Test Accuracy \\
\hline Prajapati et al. [18] & K means clusterization & $73.33 \%$ \\
\hline Proposed method & Local Threshold & $91.67 \%$ \\
\hline
\end{tabular}

Due to the best classification performance, as presented in Table 2, only DenseNet121 has been used as the CNN model for the later experiments. 10-fold cross validation has been used for UCI Machine Learning Repository and GitHub dataset and corresponding results are reported in Table 3.

To investigate the impact of segmentation of the leaves on classification performance, an experiment has been conducted on the UCI Machine Learning Repository using both segmented leaf images and non-segmented leaf images and the comparison with respect to the achieved accuracies is presented in Table 4. Accuracies for segmented leaf images are better than that for non-segmented leaf images which suggests that segmenting disease-spots in the images improves classification accuracy.

The authors of [18] have worked on classification of bacterial leaf blight, brown spot and leaf smut diseases. They proposed centroid feeding based k-means clustering for segmentation. They have achieved $73.3 \%$ classification accuracy. Result of this study has been compared with that of the proposed method in Table 5, where it is evident that the proposed method performs better.

In the final experiment, we trained the model with all the collected and captured images and achieved the test accuracy of $78.84 \%$ where the validation accuracy was $82.03 \%$. The accuracy dropped because, the images of different datasets were taken in different conditions with different types of cameras. As a result, colors of the disease-affected leaves were also different in different datasets. So, when we combine them, it creates confusion to the neural network while extracting important features.

Some important findings from these results can be beneficial for further exploration. While using CNN model for this classification problem, Resnet50 is not the right choice, rather DenseNet121 should be the used. Segmentation of the disease-affected regions in the leaf images is effective to enhance the classification accuracy. Heterogeneous sources of images have negative effect on the classification accuracy.

\section{Conclusion}

In this study, a novel method has been proposed which applies threshold-based segmentation to extract the diseaseaffected spots on the rice leaves precisely. Three different CNN models VGG16, ResNet50 and DenseNet121 have been used to classify the diseases to determine the best model for this kind of image classification problem. CNN model Resnet50 has been found not suitable and DenseNet121 has been proved best for this kind of image classification problem based on the achieved classification accuracy. Three different datasets, including our own one, have been used 
to assess the consistency of the proposed method and to verify the impact of diverse images captured in various conditions on classification performance. Negative effect on the classification accuracy has been evident for heterogeneous sources of images. Classification performance of the proposed method can still be improved. Following same image acquisition protocols while capturing images can make the images homogenous which eventually can contribute to improve the performance of the classifier. Adding more images for training can make the model more accurate and robust. Collections of images for each disease can be enriched by adding images with variations as much as possible which can enhance the robustness and consistency of the classifier. Instead of relying only on images of disease-affected leaves, weather data can also be used for rice disease classification. The use of a combination of weather data and image data to classify rice diseases can have a significant impact. Improved robustness, consistency and accuracy of this work can make it appropriate as a practical application to be used by the farmers in the field.

\section{Acknowledgement}

We acknowledge the assistance of Bangladesh Rice Research Institute (BRRI), Gazipur, Dhaka, Bangladesh for their constant support in capturing images and labeling them properly.

\section{References}

[1] Factsheet: Disease pests and management of paddy (Bacterial Leaf Blight). http://knowledgebankbrri.org/Rice_Production_Training_Manual/Day_3/Module_10/Factsheet2\%20-\%20Patapora\%20rog.pdf Accessed Date: May 23, 2021.

[2] Factsheet: Disease pests and management of paddy (Rice Blast). http://knowledgebankbrri.org/Rice_Production_Training_Manual/Day_3/Module_10/Factsheet4\%20-\%20Blast\%20rog.pdf Accessed Date: May 23, 2021.

[3] Factsheet: Disease pests and management of paddy (Sheath Blight). http://knowledgebankbrri.org/Rice_Production_Training_Manual/Day_3/Module_10/Factsheet3\%20-\%20Kholpora\%20rog.pdf Accessed Date: May 23, 2021.

[4] The global staple. http://ricepedia.org/rice-as-food/the-global-staple-rice-consumers. Accessed Date: March 24, 2020.

[5] How to manage pests and diseases. http://www.knowledgebank.irri.org/step-by-step-production/growth/pests-and-diseases. Accessed Date: March 24, 2020.

[6] Rice Leaf Diseases Data Set. https://archive.ics.uci.edu/ml/datasets/Rice Leaf Diseases. Accessed Date: March 25, 2020.

[7] M. Al-Amin, D. Z. Karim, and T. A. Bushra, "Prediction of rice disease from leaves using deep convolution neural network towards a digital agricultural system," 22nd International Conference on Computer and Information Technology (ICCIT), pp. $1-5,2019$.

[8] aldrin233 RiceDiseases-DataSet. https://github.com/aldrin233/RiceDiseases-DataSet. Accessed Date: March 25, 2020.

[9] Megha P Arakeri, Malavika Arun, Padmini R K,"Analysis of Late Blight Disease in Tomato Leaf Using Image Processing Techniques", International Journal of Engineering and Manufacturing (IJEM), Vol.5, No.4, pp.12-22, 2015.DOI: 10.5815/ijem.2015.04.02

[10] A. Asfarian, Y. Herdiyeni, A. Rauf, and K. H. Mutaqin, "Paddy diseases identification with texture analysis using fractal descriptors based on fourier spectrum," International Conference on Computer, Control, Informatics and Its Applications (IC3INA), pp. 77-81, November, 2013.

[11] S. Ghosal and K. Sarkar, "Rice leaf diseases classification using cnn with transfer learning," IEEE Calcutta Conference (CALCON), pp. 230-236, 2020.

[12] L. P. Gianessi, "Importance of pesticides for growing rice in South and South East Asia," International Pesticide Benefit Case Study, p. 108, 2014.

[13] W.-j. Liang, H. Zhang, G.-f. Zhang, and H.-x. Cao, "Rice blast disease recognition using a deep convolutional neural network," Scientific reports, Vol. 9, Is. 1, pp.1-10, 2019.

[14] B. Liu, Y. Zhang, D. He, and Y. Li, "Identification of apple leaf diseases based on deep convolutional neural networks," Symmetry, Vol. 10, Is. 1, p. 11, 2018.

[15] Y. Lu, S. Yi, N. Zeng, Y. Liu, and Y. Zhang, "Identification of rice diseases using deep convolutional neural networks," Neurocomputing, 267, pp. 378-384, 2017.

[16] D. Mohapatra, J. Tripathy, and T. K. Patra, "Rice disease detection and monitoring using cnn and naive bayes classification," Soft Computing Techniques and Applications, pp. 11-29. Springer, Singapore.

[17] F. T. Pinki, N. Khatun, and S.M. M. Islam, "Content based paddy leaf disease recognition and remedy prediction using support vector machine," 20th International Conference of Computer and Information Technology (ICCIT), pp. 1-5, December, 2017.

[18] H. B. Prajapati, J. P. Shah, and V. K. Dabhi, "Detection and classification of rice plant diseases," Intelligent Decision Technologies, Vol. 11, Is. 3, pp. 357-373, 2017.

[19] D. Singh, Asir Antony Gnana, E. Jebamalar Leavline, A. K. Abirami, and M. Dhivya. "Plant disease detection system using bag of visual words." IJ Inf Technol Comput Sci, Vol. 8, no. 9, pp. 57-63, 2018.

[20] C. R. Rahman, P. S. Arko, M. E. Ali, M. A. I. Khan, S. H. Apon, F. Nowrin, et al. "Identification and recognition of rice diseases and pests using convolutional neural networks,". Biosystems Engineering, Vol. 194, pp. 112-120, 2020.

[21] P. Sharma, Y. P. S. Berwal, and W. Ghai, "Performance analysis of deep learning cnn models for disease detection in plants using image segmentation. Information Processing in Agriculture,” Vol. 7, Is. 4, pp. 566-574, 2020.

[22] Jagadeesh D. Pujari, Rajesh.Yakkundimath, Abdulmunaf. Syedhusain. Byadgi,"Automatic Fungal Disease Detection based on Wavelet Feature Extraction and PCA Analysis in Commercial Crops", IJIGSP, vol.6, no.1, pp.24-31, 2014.DOI: 
10.5815/ijigsp.2014.01.04

[23] S. Sladojevic, M. Arsenovic, A. Anderla, D. Culibrk, and D. Stefanovic, "Deep neural networks-based recognition of plant diseases by leaf image classification". Computational intelligence and neuroscience, May, 2016.

[24] Q. Yao, Z. Guan, Y. Zhou, J. Tang, Y. Hu, and B. Yang, "Application of support vector machine for detecting rice diseases using shape and color texture features," International conference on engineering computation, pp. 79-83, May, 2009.

[25] G. Zhou, W. Zhang, A. Chen, M. He, and X. Ma, "Rapid detection of rice disease based on FCM-KM and faster R-CNN fusion," IEEE Access, vol. 7, pp. 143190-143206, 2019.

\section{Authors' Profiles}

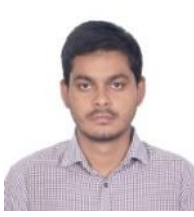

Anam Islam completed his BSc in Computer Science and Engineering from Khulna University, Bangladesh in 2020. His research interest includes, Image processing, Data analysis and Machine learning.

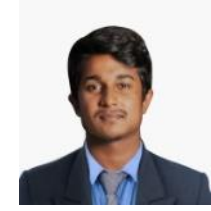

Redoun Islam is about to finish is B.sc form Khulna University in computer science. Currently his research interest includes: Machine learning for designing enemy behavior in games and implementing CNN in unity game engine.

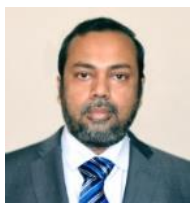

S M Rafizul Haque received PhD degree from University of Saskatchewan, Canada in 2016. He received BSc degree in Computer Science and Engineering from Khulna University, Bangladesh and MSc degree in Computer Science from Blekinge Institute of Technology, Sweden. He is a professor in the Computer Science and Engineering Discipline, Khulna University, Bangladesh. His research interests include image processing, computer vision, machine learning and data mining. He is currently working as a research scientist in the Saskatoon Laboratory of Canadian Food Inspection Agency. He has published several research papers in international journals and conferences.

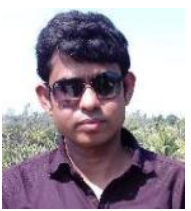

S.M. Mohidul Islam is a faculty member at the Computer Science and Engineering Discipline, Khulna University, Bangladesh. He received his B.Sc. Engg. And M.Sc. Engg. Degree from Khulna University. His research interests include Machine learning, Data Mining, Pattern Recognition, and Digital Image Processing.

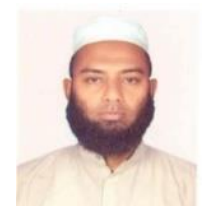

Mohammad Ashik Iqbal Khan is working as Principal Scientific Officer at Plant Pathology Division, Bangladesh Rice Research Institute (BRRI), Bangladesh. He obtained his PhD from Saga University, Japan. During his PhD, he served as Teaching Assistant for five years. Thereafter, while working at Japan International Research Center of Agricultural Science (JIRCAS) as a Post Doc. Fellow, he was able to apply many of the skills on rice blast disease resistance studies. He is the focal person of Blast Network Project in Bangladesh and maintaining collaborative research with Japan, Bangladesh, West Africa, China, Vietnam, Cambodia and Indonesia. He has served as a Research Supervisor of ten MS and five PhD students of different national and international university. He received many national and international awards for the recognition of his excellence. He served as Principal Investigator of 13 national and international projects. He has already published 62 scientific papers in national and international reputed journal and more than 50 seminar papers. $\mathrm{He}$ is involved in several e-learning and machine learning projects.

How to cite this paper: Anam Islam, Redoun Islam, S. M. Rafizul Haque, S.M. Mohidul Islam, Mohammad Ashik Iqbal Khan, "Rice Leaf Disease Recognition using Local Threshold Based Segmentation and Deep CNN", International Journal of Intelligent Systems and Applications(IJISA), Vol.13, No.5, pp.35-45, 2021. DOI: 10.5815/ijisa.2021.05.04 